\title{
Síndrome da disfunção cognitiva canina: revisão de literatura
}

\section{Canine cognitive dysfunction syndrome: literature review}

\author{
Bruna Carvalho da Silva ${ }^{1}$, Beatriz Gneiding ${ }^{2 *}$, Joelma Lucioli ${ }^{1}$, Jéssica Sara Tesser ${ }^{1}$, José Eduardo Basilio de \\ Oliveira Gneiding ${ }^{1,2}$ \\ ${ }^{1}$ Universidade Regional de Blumenau (FURB), Blumenau, SC, Brasil \\ 2 Pontifícia Universidade Católica do Paraná (PUCPR), Curitiba, PR, Brasil
}

\section{Resumo}

0 presente trabalho teve por objetivo dissertar sobre os aspectos atuais acerca do processo de envelhecimento, bem como da síndrome da disfunção cognitiva canina. 0 processo de envelhecimento cerebral nos cães se caracteriza por alterações como atrofia cortical, mineralização meníngea, lesões perivasculares, angiopatia amiloide cerebrovascular, perda de neurônios, desmielinização, acúmulo de placas senis, danos oxidativos, entre outras. Devido à degeneração patológica que ocorre no cérebro, as alterações comportamentais são os primeiros sinais de declínio cognitivo em cães geriátricos. Desse modo, a síndrome da disfunção cognitiva (SDC) é reconhecida como uma doença neurodegenerativa que acomete animais a partir dos 6 anos de idade, sendo seriamente subdiagnosticada, pois os tutores omitem as mudanças comportamentais de seus animais nas consultas veterinárias, acreditando que estas são alterações normais relacionadas ao envelhecimento. Outro fator importante é a falta de questionamento dos médicos veterinários quanto a mudanças que indicam declínio cognitivo. 0 diagnóstico é complexo e pode envolver exames de imagem, laboratoriais, aplicação de questionários específicos para alterações comportamentais, além da exclusão de outras enfermidades. 0 tratamento consiste na utilização de fármacos, enriquecimento ambiental, alteração no manejo e na dieta, objetivando retardar a evolução da doença e melhorar a qualidade de vida do paciente.

Palavras-chave: Geriatria. Cães. Cognição. Senescência. Neurologia.

\begin{abstract}
The aim of the present study was to discuss current aspects about the aging process as well as the canine cognitive dysfunction syndrome. The aging process in dogs is characterized by alterations such as cortical atrophy, meningeal mineralization, perivascular lesions, cerebrovascular amyloid angiopathy, loss of neurons, demyelination, accumulation of senile plaques,
\end{abstract}


oxidative damage, among others. Due to the pathological degeneration that occurs in the brain, behavioral changes are the first signs of cognitive decline in geriatric dogs. Thus cognitive dysfunction syndrome (CDS) is recognized as a neurodegenerative disease that affects animals from the age of six, being seriously underdiagnosed, since the tutors omit the behavioral changes of their animals in veterinary consultations believing that these are normal changes related to aging. Another important factor is the lack of questioning of veterinarians about changes that indicate cognitive decline. The diagnosis is complex and it may involve image and laboratory tests, application of specific questionnaires for behavioral changes besides the exclusion of other diseases. The treatment consists in the use of drugs, environmental enrichment, alteration in management and diet, aiming to delay the evolution of the disease and improve the quality of life of the patient.

Keywords: Geriatrics. Dogs. Cognition. Aging. Neurology.

\section{Introdução}

Com o atual estilo de vida das pessoas e a nova composição das famílias, houve um crescente avanço no relacionamento entre os seres humanos e os animais domésticos, passando estes a ocupar um lugar de destaque no ambiente familiar. Este fator, aliado ao zelo e dedicação dos tutores, desenvolvimento de novos métodos de diagnóstico, medicamentos e tratamentos, contribui para o aumento da expectativa de vida dos animais de companhia e, desta forma, o atendimento a cães idosos se tornou mais frequente na rotina clínica (Fortney, 2008; Ferreira e Sampaio, 2010).

0 processo de envelhecimento nos cães apresenta características evidentes, alterações celulares progressivas e irreversíveis, podendo ocasionar mudanças nas condições fisiológicas, resultando em distúrbios médicos como doenças degenerativas, deterioração neurológica, declínio sensorial e mudanças de comportamento, sendo a última constantemente o primeiro ou até mesmo o único sinal de doença nos cães senis, podendo estar relacionada a dor e/ou declínio cognitivo. Neste quadro se destaca a síndrome da disfunção cognitiva (SDC), por ser considerada uma das principais doenças neurodegenerativas progressivas, que se caracteriza pelo declínio cognitivo e consequente desvios comportamentais (Landsberg e Araujo, 2005; Osella et al., 2007; Babicsak e Vulcano, 2013).

De acordo com Boulard (2005) e Laflamme (2012), os primeiros sinais clínicos de enfermidades senis em cães se manifestam a partir dos 7 anos, o que torna imprescindível a realização de criteriosa avaliação clínica destes pacientes, pois podem apresentar variadas condições médicas com diferentes graus de disfunção orgânica (Head et al., 2008; Vite e Head, 2014). Tendo em vista este fato, o presente trabalho teve por objetivo dissertar sobre os aspectos atuais acerca do processo de envelhecimento canino, bem como da síndrome da disfunção cognitiva canina.

\section{Processo de envelhecimento}

Devido às modificações fisiológicas que os cães idosos apresentam, observam-se alterações no sistema nervoso, tais como atrofia cortical, aumento do tamanho ventricular, estreitamento dos giros, dilatação dos sulcos, espessamento da leptomeninge (aracnoide e pia-máter), mineralização meníngea, lesões perivasculares, angiopatia amiloide cerebrovascular, perda de neurônios, desmielinização, acúmulo de placas senis e danos oxidativos (Borràs et al., 1999; Landsberg et al., 2004; Briones et al., 2010; Vite e Head, 2014).

Com o objetivo de observar alterações que o envelhecimento desenvolve no cérebro, Tapp et al. (2004) utilizaram em seu estudo 66 cães da raça Beagle, com idades entre 3 meses e 15 anos de idade, e avaliaram por meio de ressonância magnética a ação da idade no volume do lobo frontal. Nos indivíduos entre 6 meses e 7 anos, o volume deste lobo se manteve estável, entretanto, a partir dos 8 anos houve significativa diminuição, além de decréscimo do hipocampo e aumento dos ventrículos no córtex frontal. Já os animais com 12 anos ou mais apresentaram ainda redução do volume total do cérebro. Desta forma, os pesquisadores concluíram que o lobo frontal e o hipocampo são regiões sensíveis ao envelhecimento e a atrofia do lobo frontal ocorre nos primeiros anos de senescência devido a 
alterações neuropatológicas nesta área. A redução do parênquima cortical leva, consequentemente, ao aumento dos ventrículos intracranianos, sendo também comumente identificado o espessamento da leptomeninge. Todavia, acredita-se não haver relação desta com o desenvolvimento dos sinais clínicos neurológicos. Também em cães de raças grandes, a partir dos 2 anos de idade já se pode observar placas de ossificação na dura-máter (Tapp et al., 2004; Fortney, 2008; Ecco et al., 2016).

Além das alterações morfológicas e estruturais, pode-se observar também o aumento da produção de radicais livres em cães senis, pois, metabolicamente, parte do oxigênio utilizado para a produção de energia pelas mitocôndrias resulta em espécies reativas do mesmo. Por mais que existam enzimas e vitaminas que possam inativar estes agentes oxidantes, à medida que o animal envelhece este sistema de proteção tende a se tornar falho. Quando na grande exposição aos radicais livres, a reação junto às proteínas e lipídeos causa alterações como mutações e morte celular. Em adição, a continuidade desta situação propicia o desenvolvimento de processos inflamatórios e de infecções oportunistas, levando à consequente disfunção neural e/ou morte de neurônios (Ferreira e Matsubara, 1997; Bartzokis et al., 2004; Landsberg et al., 2004; Rofina et al., 2006; Youssef et al., 2016).

Do mesmo modo, o processo de envelhecimento também é caracterizado pelo acúmulo de proteínas dentro e ao redor dos neurônios, que passam a se tornar tóxicas. Assim, um achado post mortem frequente em cérebros de cães geriátricos são as placas senis, constituídas por agregados moleculares extracelulares de presenilina 1 (PS1), apolipoproteína E (apoE) e $\beta$-amiloide $(A \beta)$, sendo o último o componente primário. Estes advêm da proteína precursora de amiloide (APP), que é secretada pelos neurônios no espaço interneural, concentrando-se no cérebro (Dimakopoulos e Mayer, 2002; Head et al., 2008; Davis e Head, 2014). Nos cérebros caninos, as deposições das placas senis ocorrem em diferentes idades. Inicialmente, forma-se o acúmulo no córtex préfrontal (aproximadamente aos 9 anos de idade), córtex temporal, hipocampo e córtex occipital, respectivamente. 0 depósito de proteína $A \beta$ está associado com deficit nas sinapses, danificação da atividade neural, apoptose de neurônios e esgotamento de neurotransmissores. Em razão da proteína $A \beta$ ser $98 \%$ homóloga em cães e seres humanos, surgiu o consequente interesse na espécie canina como modelo para estudos sobre a doença de Alzheimer (Head et al., 2000; Landsberg et al., 2004; Youssef et al., 2016).

Além de formar as placas senis, a proteína $A \beta$ é a mais observada na angiopatia amiloide cerebral (CAA). Ela se deposita na parede das arteríolas corticais e leptomeningeais, capilares e vasos, promovendo a degeneração da parede vascular, comprometendo a integridade da barreira hematoencefálica e ocasionando micro-hemorragias que atingem de forma mais grave o lobo occipital. A CAA pode também prejudicar o fluxo sanguíneo e a utilização da glicose (Rensink et al., 2003; Yamada, 2004; Rofina et al., 2006; Chapagain et al., 2017).

\section{Síndrome da disfunção cognitiva (SDC)}

Reconhecida pelo declínio cognitivo progressivo devido à degeneração patológica do cérebro, a SDC é uma doença neurodegenerativa que acomete animais idosos e se caracteriza pelas alterações de comportamento. Esta afecção impacta a qualidade de vida do cão e sua relação com o tutor, pois as mudanças comportamentais prejudicam o vínculo entre ambos e, nos casos mais graves, pode ser a causa da opção pela eutanásia (Bennett, 2012; Marcolino et al., 2016).

\section{Prevalência}

A partir dos 6 anos de idade os animais já podem apresentar alterações comportamentais discretas, no entanto os sinais são mais consistentes por volta dos 11 anos de idade. Entre os 7 e os 9 anos, a prevalência da doença é de $20 \%$ a $30 \%$, e aumenta para 66\% nos cães com mais de 14 anos (Laflamme, 2012; Marcolino et al., 2016).

Em estudo epidemiológico conduzido por Salvin et al. (2010), que objetivou estimar a prevalência de SDC em cães, bem como determinar a taxa de animais diagnosticados anteriormente, os autores concluíram que $14,2 \%$ dos pacientes analisados apresentavam sinais sugestivos de SDC, no 
entanto, apenas $1,9 \%$ destes já haviam recebido o diagnóstico anteriormente. A prevalência da doença no mundo é desconhecida, mas com base em estudos realizados na Austrália, Estados Unidos e Reino Unido, as estimativas estão entre 14 e 30\% (Oliveira et al., 2016).

\section{Subdiagnóstico}

As alterações comportamentais são frequentemente os primeiros sinais clínicos de declínio cognitivo, sendo desafiador diagnosticar precocemente a DC, tendo em vista a pluralidade de fatores para modificações comportamentais. Não obstante, a omissão por parte dos tutores em relatar aos médicos veterinários os eventuais distúrbios comportamentais que seus animais senis e geriátricos apresentam dificulta ainda mais esta situação (Landsberg et al., 2004; Landsberg e Araujo, 2005; Landsberg et al., 2011). Possivelmente, esta omissão se deve ao fato de acreditarem que este tipo de informação é irrelevante para a discussão durante as consultas e por atribuírem essas alterações ao processo natural do envelhecimento dos cães. Portanto, para o devido acompanhamento de animais idosos, o esclarecimento acerca da DC é uma conduta importante a ser adotada pelo clínico.

Além da responsabilidade do tutor, o médico veterinário deve ser proativo nas estratégias adotadas durante a anamnese, visto que as alterações do comportamento podem ser sutis (Landsberg e Ruehl, 1997; Landsberg et al., 2011; Davies, 2012). Osella et al. (2007) conseguiram explicitar tal situação realizando uma pesquisa sobre a prevalência de sinais clínicos de SDC em um grupo de cães geriátricos, no qual os tutores afirmavam desconhecer os distúrbios de comportamento em seus animais. Os autores concluíram que dos 124 cães analisados por meio de questionário específico, 75 deles apresentaram sinais sugestivos de disfunção cognitiva.

\section{Sinais Clínicos}

Os efeitos do envelhecimento sobre o cérebro são os responsáveis pelas mudanças comportamentais que caracterizam a SDC. Entre os sinais observados no cão estão confusão, alterações nas relações sociais com as pessoas (membros da família) e outros animais, apatia, ansiedade, alterações no ciclo de sono-vigília, problemas de aprendizagem e memória, alterações de apetite e aumento da irritabilidade (Gallego et al., 2010; Landsberg et al., 2012; Svicero et al., 2017)

Os sinais clínicos são definidos também de forma mais ampla em quatro categorias: (1) desorientação, (2) alteração na interação com os tutores e outros animais, (3) alterações no ciclo de sono-vigília e (4) sujeira doméstica. Alguns autores propõem mais uma categoria: (5) redução das atividades (Landsberg e Ruehl, 1997; Landsberg et al., 2004; Svicero et al., 2017).

\section{Diagnóstico}

O diagnóstico definitivo é confirmado apenas no exame neuropatológico post mortem do cérebro. Entretanto, o exame presuntivo pode ser realizado com base na anamnese, exame neurológico, exames complementares, por exclusão de outras doenças, exames neuropsicológicos e aplicação de um questionário específico para alterações de comportamento (Bagley, 1997; Svicero et al., 2017).

Durante a anamnese é importante perguntar ao tutor se o animal está utilizando algum tipo de medicamento, pois alguns fármacos podem provocar como efeitos adversos desde alterações comportamentais até mudanças fisiológicas, como poliúria e polifagia. Dentre os exames complementares a serem solicitados estão hemograma, perfil bioquímico renal e hepático, dosagens hormonais e análise do líquido cérebroespinhal para descartar a possibilidade de patologias que provoquem o aumento da pressão intracraniana e doenças inflamatórias. Os exames de imagem são úteis para identificar alterações intracranianas, e para esta finalidade os mais indicados são a tomografia computadorizada e a ressonância magnética (Landsberg et al., 2012; Oliveira et al., 2016).

A exclusão de outras doenças que possam mimetizar os sinais clínicos de SDC é imprescindível e determinante para o diagnóstico correto, já que neoplasias intracranianas, encefalopatia hepática, cardiopatia, hipotireoidismo e outras doenças que causam dor podem provocar mudanças de 
comportamento similares às observadas em cães com disfunção cognitiva (Landsberg e Araujo, 2005).

Os exames neuropsicológicos permitem uma avaliação objetiva e quantitativa dos deficits de aprendizagem e memória. Para esta avaliação, aplicase em laboratório uma série de testes que buscam investigar as alterações consistentes com a doença, no entanto, o que impede sua realização em consultas de rotina são a sua aplicabilidade. Desse modo, o que se tornou viável para aplicar ao tutor na rotina clínica foi o questionário específico para alterações comportamentais conforme proposto por Landsberg et al. (2004), ainda utilizado atualmente (Landsberg e Araujo, 2005; Davis e Head, 2014; Svicero et al., 2017). Estes questionários se mostraram eficazes para diagnosticar SDC clinicamente, no entanto, devem abranger uma quantidade ampla de perguntas que investiguem o comportamento do paciente e precisam ser aplicados por clínicos ou pesquisadores, pois, quando entregues aos tutores, pode haver equívocos na interpretação das questões e alterações sutis podem ser omitidas (Landsberg et al., 2011; Svicero et al., 2017).

A formulação dos questionários inclui, em média, 32 perguntas que se dividem em oito categorias, descritas na Tabela 1.

Tabela 1 - Subdivisão das oito categorias que englobam as questões contidas no questionário utilizado como ferramenta para o diagnóstico da síndrome da disfunção cognitiva em cães

\begin{tabular}{l|l}
\hline A & Confusão - Consciência - Orientação espacial \\
B & Relações - Comportamentos sociais \\
C & Atividade - Aumentada/repetitiva \\
D & Atividade - Reduzida/apatia \\
E & Ansiedade - Aumento da irritabilidade \\
F & Ciclos de sono-vigília - Reversão do esquema dia/noite \\
G1 & Aprendizado e memória - Sujeira doméstica \\
G2 & Aprendizado e memória - Trabalho, tarefas e comandos \\
\hline
\end{tabular}

Nota: Modificado de Landsberg et al. (2004).

Utilizando este questionário comportamental, Marcolino et al. (2016) avaliaram em seu trabalho as funções cognitivas de 30 cães com mais de 8 anos de idade e observaram que 93,33\% dos animais apresentavam alterações em duas ou mais categorias.

Por meio desta série de perguntas é possível detectar mudanças discretas do comportamento que podem indicar os primeiros sinais clínicos de declínio cognitivo. Lembrando que quanto antes este distúrbio for diagnosticado, melhor será o prognóstico.

\section{Tratamento}

Apesar da SDC não ter cura, o objetivo da terapia é retardar a evolução da enfermidade, de forma que o processo degenerativo evolua lentamente, atenuando os sinais clínicos precocemente. 0 tratamento indicado para cães com SDC é o enriquecimento ambiental, utilização de medicamentos, dietas suplementadas com antioxidantes e mudanças no manejo dos animais (Milgram et al., 2005; Landsberg et al., 2012). 0 enriquecimento ambiental inclui novos truques e brincadeiras, passeios, atividade física, entre outras estratégias para estimular a cognição do animal; esses exercícios auxiliam, ainda, no ciclo de sono-vigília. 0 objetivo do tratamento farmacológico consiste na restauração dos níveis próximos da normalidade dos neurotransmissores, evitando a rápida progressão da neurodegeneração; dessa forma, medicamentos ansiolíticos como alprazolam ou diazepam são utilizados a fim de manter o animal tranquilo durante a noite. Para os cães que apresentam distúrbios do sono, pode-se administrar melatonina cerca de 30 minutos antes de o animal dormir (Landsberg et al., 2011; Oliveira et al., 2016).

A selegilina também é um fármaco indicado para o tratamento da SDC. Ela aumenta os níveis de dopamina, reduz o risco de morte celular e também desenvolve a síntese de fatores de crescimento para nervos, além de possuir efeito antioxidante. Todas essas ações ocorrem devido à inibição seletiva e irreversível da enzima monoamina-oxidase $\mathrm{B}$ (MAO-B). Este medicamento tem demostrado resultados satisfatórios para o tratamento da SDC, atenuando os sinais clínicos da doença (Gallego et al., 2010).

Outra alternativa terapêutica é a propentofilina, recomendada por possuir um efeito neuroprotetor, melhorando o fluxo sanguíneo no sistema nervoso. 
Já a nicergolina melhora a utilização da glicose pelo cérebro, eleva a biossíntese de ácidos nucleicos e proteínas, além de possuir ação em sistemas de neurotransmissão. Fármacos como adrafinil e modafinil também estão sendo propostos devido a sua ação potencializadora no sistema noradrenérgico (Azkona et al., 2005; Gallego et al., 2010; Bondan et al., 2016). No entanto, ao instituir o tratamento para SDC em cães, deve-se considerar o estado de saúde geral do paciente, assim como a utilização de outras medicações já administradas ao animal, pois estes fármacos podem causar efeitos adversos ou interações medicamentosas, colocando em risco o tratamento ou a vida do paciente (Landsberg et al., 2011).

Os suplementos nutricionais também são utilizados na terapia da doença. Sabendo-se que a produção de espécies reativas de oxigênio atua diretamente no processo de envelhecimento cerebral, causando declínio da cognição, esses suplementos melhoram os sistemas antioxidantes, reduzindo a formação das espécies reativas de oxigênio (Osella et al., 2007). Dentre os produtos utilizados nesta suplementação estão vitamina C e E, selênio, betacaroteno, L-carnitina e ácido alfa-lipóico. Outras alternativas incluem o uso de gingko biloba, ácidos graxos e ingestão de frutas e vegetais (Gallego et al., 2010; Neves et al., 2010; Oliveira et al., 2016).

A medicina veterinária integrativa tem sido utilizada para o tratamento de doenças neurológicas e tem demonstrado resultados bastante satisfatórios; estas representam, juntamente com as doenças musculoesqueléticas, $70 \%$ dos casos encaminhados para acupuntura. Já se sabe que a aplicação da acupuntura possui ação em estruturas cerebrais como o córtex (Scognamillo-Szabó e Bechara, 2010; Collazo, 2012).

Em relato de Campos et al. (2010), que objetivou avaliar pacientes de um setor homeopático e analisar a distribuição das principais doenças, bem como a relação dos medicamentos prescritos, os autores observaram que as afecções comportamentais foram uma das principais causas da procura do serviço. Ainda, grande parte dos animais avaliados eram idosos, evidenciando a maior qualidade de vida que estão tendo devido aos avanços na medicina veterinária e aos cuidados por parte dos tutores.

\section{Conclusão}

Devido à nova forma de relação que se instituiu entre animais e seres humanos, e aos avanços na medicina veterinária, a expectativa de vida dos cães está aumentando gradativamente. Considerando que a partir dos 6 anos de idade os cães podem começar a demonstrar os primeiros sinais clínicos de alterações relacionadas com a senilidade, a observação minuciosa das mudanças comportamentais dos animais é de extrema importância no diagnóstico precoce da SDC.

Assim que o diagnóstico for realizado, a definição do tratamento deve ser prontamente efetuada e, para o sucesso deste, o tutor deve estar disposto a fazê-lo adequadamente, pois além da administração de medicamentos, podem ser necessárias alterações na dieta e no manejo do animal. Desse modo, os resultados positivos podem ser observados brevemente, melhorando a qualidade de vida do cão e sua relação com o tutor.

\section{Referências}

Azkona G, Garcia-Belenguer S, Unzueta A, Chacón G, Rosado B, León M, et al. Tratamiento con nicergolina de dos casos de disfunción cognitiva en perros geriátricos. Clin Vet Peq Anim. 2005;25(1):11-5.

Babicsak VR, Vulcano LC. Alterações senis do encéfalo de caninos: Aspectos imaginológicos. Vet e Zootec. 2013;20(2):238-49.

Bagley RS. Common neurologic diseases of older animals. Vet Clin North Am Small Anim Pract. 1997; 27(6):1451-86.

Bartzokis G, Sultzer D, Lu PH, Nuechterlein KH, Mintz J, Cummings JL. Heterogeneous age-related breakdown of white matter structural integrity: implications for cortical "disconnection" in aging and Alzheimer's disease. Neurobiol Aging. 2004;25(7):843-51.

Bennett S. Cognitive dysfunction in dogs: pathologic neurodegeneration or just growing older? Vet J. 2012;194(2):141-2. 
Bondan EF, Martins MFM, Dossa PD, Viebig LB, Cardoso $\mathrm{CV}$, Martins Jr JL, et al. Propentofylline reduces glial scar development following gliotoxic damage in the rat brainstem. Arq Neuropsiquiatr. 2016;74(9):730-6.

Borràs D, Ferrer I, Pumarola M. Age-related changes in the brain of the dog. Vet Pathol. 1999;36(3):202-11.

Boulard G. Grow Old With Me. 2005 [acesso 20 mar 2018]. Disponível em: https://tinyurl.com/y88t75z8.

Briones F, Cáceres T, Jarpa M. Detección de b-Amiloide, Proteína TAU Hiperfosforilada y Ubiquitina por Técnica de Inmunohistoquímica en Cerebros de Caninos Mayores de 10 Años. Int J Morphol. 2010;28(4):1255-61.

Campos FL, Benin LA, Camargo VMF. Perfil do atendimento em homeopatia da Clínica Escola Veterinária (CEVET) da Universidade Estadual do Centro-Oeste (UNICENTRO), Paraná, Brasil. Ambiencia. 2010;6(2):289-96.

Chapagain D, Virányi Z, Wallis LJ, Huber L, Serra J, Range F. Aging of attentiveness in Boder Collies and other pet dog breends: the protective benefits of lifelong training. Front Aging Neurosci. 2017;9:100.

Collazo E. Fundamentos actuales de la terapia acupuntural. Rev Soc Esp Dolor. 2012;19(6):325-31.

Davies M. Geriatric screening in first opinion practice - results from 45 dogs. J Small Anim Pract. 2012;53(9):507-13.

Davis PR, Head E. Prevention approaches in a preclinical canine model of Alzheimer's disease: benefits and challenges. Front Pharmacol. 2014;5:1-14.

Dimakopoulos AC, Mayer RJ.Aspects of neurodegeneration in the canine brain. J Nutr. 2002;132(6 Supl 2):S1579-82.

Ecco R, Viott AM, Graça DL, Alessi AC. Sistema Nervoso. In: Santos RL, Alessi AC. Patologia Veterinária. 2 ed. Rio de Janeiro: Roca; 2016. p. 487-572.

Ferreira ALA, Matsubara LS. Radicais livres: conceitos, doenças relacionadas, sistema de defesa e estresse oxidativo. Rev Assoc Med Bras. 1997;43(1):61-8.
Ferreira SA, Sampaio IBM. Relação homem-animal e bemestar do cão domiciliado. Arch Vet Sci. 2010;15(1):22-35.

Fortney WD. Geriatria e Senilidade. In: Hoskins JD. Geriatria e gerontologia do cão e gato. 2 ed. São Paulo: Roca; 2008. p. 1-4.

Gallego D, Figueroa J, Orozco C. Síndrome de disfunción cognitiva de perros geriátricos. Rev MVZ Cordoba. 2010;15(3):2252-62.

Head E, McCleary R, Hahn FF, Milgram NW, Cotman CW. Region-specific age at onset of beta-amyloid in dogs. Neurobiol Aging. 2000;21(1):89-96.

Head E, Rofina J, Zicker S. Oxydative stress, aging, and central nervous system disease in the canine model of human brain aging. Vet Clin North Am Small Anim Pract. 2008;38(1):167-78.

Laflamme DP. Nutritional care for aging cats and dogs. Vet Clin North Am Small Anim Pract. 2012;42(4):769-91.

Landsberg G, Araujo JA. Behavior problems in geriatric pets. Vet Clin North Am Small Anim Pract. 2005;35(3):675-98.

Landsberg GM, Hunthausen W, Ackerman L. Problemas comportamentais do cão e do gato. 2 ed. São Paulo: Roca; 2004. 492 p.

Landsberg GM, Deporter T, Araujo JA. Clinical signs and management of anxiety, sleeplessness, and cognitive dysfunction in the senior pet. Vet Clin North Am Small Anim Pract. 2011;41(3):565-90.

Landsberg GM, Nichol J, Araujo JA. Cognitive dysfunction syndrome. a disease of canine and feline brain aging. Vet Clin North Am Small Anim Pract. 2012;42(4):749-68.

Landsberg G, Ruehl W. Geriatric behavioral problems. Vet Clin North Am Small Anim Pract. 1997;27(6):1537-59.

Marcolino G, Escodro PB, Souza FW, Notomi MK. Utilização de formulário clínico para reconhecimento de distúrbio cognitivo em cães idosos. Cienc Vet Trop. 2016;19(2):11-8. 
Milgram NW, Head E, Zicker SC, Ikeda-Douglas CJ, Murphey $\mathrm{H}$, Muggenburg B, et al. Learning ability in aged beagle dogs is preserved by behavioral enrichment and dietary fortification: a two-year longitudinal study. Neurobiol Aging. 2005;26(1):77-90.

Neves IV, Tudury EA, Costa RC. Fármacos utilizados no tratamento das afecções neurológicas de cães e gatos. Semina Cienc Agrar. 2010;31(3):745-66.

Oliveira HEV, Marcasso RA, Arias MVB. Doenças cerebrais no cão idoso. Rev Cient Med Vet Pequenos Anim Anim Estim. 2016;12(45):1-15.

Osella MC, Re G, Odore R, Girardi C, Badino P, Barbero $\mathrm{R}$, et al. Canine cognitive dysfunction syndrome: Prevalence, clinical signs and treatment with a neuroprotective nutraceutical. Appl Anim Behav Sci. 2007;105(4):297-310.

Rensink AAM, de Waal RM, Kremer B, Verbeek MM. Pathogenesis of cerebral amyloid angiopathy. Brain Res Rev. 2003;43(2):207-23.

Rofina JE, van Ederen AM, Toussaint MJ, Secràve M, van der Spek A, van der Meer I, et al. Cognitive disturbances in old dogs suffering from the canine counterpart of Alzheimer's disease. Brain Res. 2006;1069(1): 216-26.
Salvin HE, McGreevy PD, Sachdev PS, Valenzuela MJ. Under diagnosis of canine cognitive dysfunction: a cross-sectional survey of older companion dogs. Vet J. 2010;184(3):277-81.

Scognamillo-Szabó MVR, Bechara GH. Acupuntura: histórico, bases teóricas e sua aplicação em Medicina Veterinária. Cienc Rural. 2010;40 (2):491-500.

Svicero DJ, Heckler MCT, Amorim RM. Prevalence of behavioral changes in senile dogs. Cienc Rural. 2017;47(2): e20151645.

Tapp PD, Siwak CT, Gao FQ, Chiou JY, Black SE, Head E, et al. Frontal lobe volume, function, and $\beta$-amyloid pathology in a canine model of aging. J Neurosci. 2004;24(38):8205-13.

Vite $\mathrm{CH}$, Head E. Aging in the canine and feline brain. Vet Clin North Am Small Anim Pract. 2014;44(6):1113-29.

Yamada M. Cerebral amyloid angiopathy and gene polymorphisms. J Neurol Sci. 2004;226(1-2):41-4.

Youssef SA, Capucchio MT, Rofina JE, Chambers JK, Uchida $\mathrm{K}$, Nakayama $\mathrm{H}$, et al. Pathology of the Aging Brain in Domestic and Laboratory Animals, and Animal Models of Human Neurodegenerative Diseases. Vet Pathol. 2016;53(2):327-48. 